\title{
Fabrication, appraisal, and transdermal permeation of sildenafil citrate-loaded nanostructured lipid carriers versus solid lipid nanoparticles
}

This article was published in the following Dove Press journal:

International Journal of Nanomedicine

5 December 2011

Number of times this article has been viewed

\author{
Yosra SR Elnaggar' \\ Magda A El-Massik ${ }^{2}$ \\ Ossama Y Abdallah' \\ 'Department of Pharmaceutics, \\ Faculty of Pharmacy, Alexandria \\ University, ${ }^{2}$ Department of \\ Pharmaceutics, Faculty of Pharmacy \\ and Drug Manufacturing, Pharos \\ University, Alexandria, Egypt
}

\begin{abstract}
Although sildenafil citrate (SC) is used extensively for erectile dysfunction, oral delivery of SC encounters many obstacles. Furthermore, the physicochemical characteristics of this amphoteric drug are challenging for delivery system formulation and transdermal permeation. This article concerns the assessment of the potential of nanomedicine for improving SC delivery and transdermal permeation. SC-loaded nanostructured lipid carriers (NLCs) and solid lipid nanoparticles (SLNs) were fabricated using a modified high-shear homogenization technique. Nanoparticle optimization steps included particle size analysis, entrapment efficiency (EE) determination, freeze-drying and reconstitution, differential scanning calorimetry, in vitro release, stability study and high-performance liquid chromatography analysis. Transdermal permeation of the nanocarriers compared with SC suspension across human skin was assessed using a modified Franz diffusion cell assembly. Results revealed that SLNs and NLCs could be optimized in the nanometric range (180 and $100 \mathrm{~nm}$, respectively) with excellent EE $(96.7 \%$ and $97.5 \%$, respectively). Nanoparticles have significantly enhanced in vitro release and transdermal permeation of SC compared with its suspensions. Furthermore, transdermal permeation of SC exhibited higher initial release from both SLN and NLC formulations followed by controlled release, with promising implications for faster onset and longer drug duration. Nanomedicines prepared exhibited excellent physical stability for the study period. Solid nanoparticles optimized in this study successfully improved SC characteristics, paving the way for an efficient topical Viagra ${ }^{\circledR}$ product.
\end{abstract}

Keywords: nanomedicine, transdermal drug delivery, erectile dysfunction, Viagra ${ }^{\circledR}$

\section{Introduction}

Erectile dysfunction has been defined as the inability to attain and/or maintain a penile erection sufficient for satisfactory sexual performance. Although erectile dysfunction represents a major clinical problem, medical therapy for this condition was invasive or ineffective before the introduction of sildenafil citrate (SC). ${ }^{1} \mathrm{SC}$ is the first oral treatment to have had significant success in the treatment of erectile dysfunction. It acts via selective inhibition of the enzyme phosphodiesterase type 5. The physiologic mechanism of penile erection involves release of nitric oxide in the corpora cavernosa during sexual stimulation. This release activates the enzyme guanylate cyclase, relaxing the smooth muscle of the corpus cavernosum and allowing greater blood flow, thus leading to an erection. ${ }^{2,3} \mathrm{SC}$ is an amphoteric drug with a $\mathrm{pH}$-dependent solubility. The drug possesses a basic functional group (NH-piperazine), characterized by a $\mathrm{pK}$ value of 8.7 , in addition to a weak acidic moiety (HN-amide), as shown in Figure 1.
Correspondence: Yosra SR Elnaggar Department of Pharmaceutics, Faculty of Pharmacy, Alexandria University, I Khartoum Square, Azarita, Messalla Post Office, PO Box 21521, Alexandria, Egypt Tel +2034870359

Fax +2034873273

Email yosra_pharm@yahoo.com 


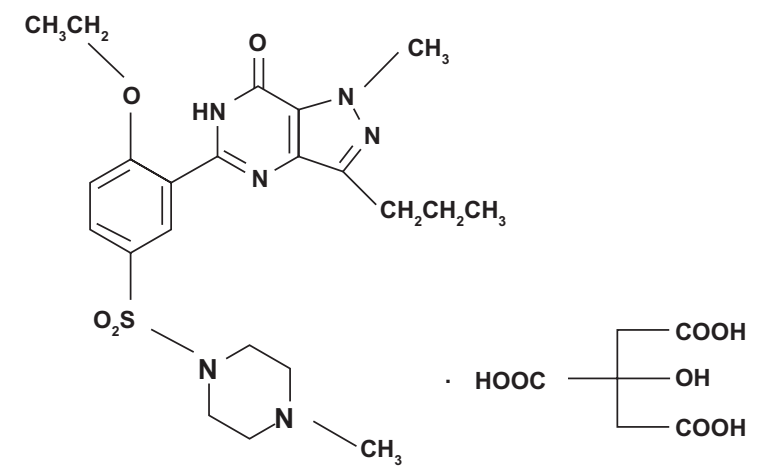

Figure I Chemical structure of sildenafil citrate.

$\mathrm{SC}$ is marketed as Viagra ${ }^{\circledR}$ oral tablets, with doses of 25,50 , and $100 \mathrm{mg}$ taken not more than once a day. ${ }^{4}$

Unfortunately, oral administration of SC encounters numerous obstacles. The drug is vulnerable to considerable intestinal and hepatic first-pass metabolism (approximately $71 \%$ of oral dose). ${ }^{5}$ The drug is metabolized in the liver by the enzyme cytochrome P3A4, with an oral bioavailability of $40 \%$. Furthermore, numerous side effects such as blood pressure reduction, headaches, flushing, and nasal congestion are concomitant with oral administration. Patients with moderate to severe cardiovascular disease or those undergoing nitrate therapy are at increased risk for potentially serious adverse cardiovascular side effects with SC therapy. In addition, relatively delayed onset (30-45 minutes) and short duration of action have been demonstrated for SC, with a half-life of 4 hours. In the meantime, repeated doses are required to sustain drug plasma levels. ${ }^{6,7}$

Transdermal delivery of SC through a local tissue area could be considered as an alternative to the oral route, in order to avoid these adverse side effects, to shorten onset time, and to sustain the effect for longer periods. ${ }^{8}$ In this context, nanosized colloidal carriers have been the subject of widespread interest in the field of transdermal drug delivery. ${ }^{8}$ Among these, solid lipid nanoparticles (SLNs) and nanostructured lipid carriers (NLCs) are attracting major attention as novel colloidal nanocarriers for topical use. Compared with liposomes and polymeric nanoparticles, SLNs exhibit the peculiarity of higher stability, capability of large-scale application, and avoidance of organic solvent incorporation. ${ }^{9,10}$ Compared with other topical vehicles such as creams, tinctures, and emulsions, combined advantages of SLNs include controlled release, negligible skin irritation, and protection of active compounds. ${ }^{7}$ Enhanced topical permeation via SLNs and NLCs is well recognized in the literature. ${ }^{11,12}$ Drugs that have exhibited improved transdermal penetration when incorporated into SLNs include flurbiprofen, ${ }^{13}$ ascorbyl palmitate,,${ }^{14}$ and ketorolac. ${ }^{15}$ On the other hand, NLCs are the second generation of SLNs incorporating a solid matrix that entraps variable liquid lipid nanocompartments. NLCs have been shown to possess improved drug loading, adjusted drug release, and minimum drug expulsion during storage. ${ }^{16}$ NLCs remain in their solid state through controlling the liquid lipid content added to the formulation; therefore, controlled drug release properties can be achieved with NLCs. ${ }^{17}$ NLCs have also proved effective in the field of transdermal drug permeation. ${ }^{18}$

Nevertheless, a general challenge facing transdermal delivery of drugs is low skin permeation; reasons for this include the physicochemical properties of the compound, low partition ability, and formidable barrier properties of the stratum corneum. The majority of drugs do not appear to penetrate the skin at a sufficiently high rate to show therapeutic effectiveness. The feasibility of the transdermal route is thus limited to powerful actives presenting features such as appropriate low molecular weight and high lipophilicity. ${ }^{8}$ Further challenges in delivery-system formulation and transdermal permeation of SC are the amphoteric nature and $\mathrm{pH}$-dependent characteristics of the compound. The drug possesses poor solubility in both aqueous and oil phases. ${ }^{4}$ Furthermore, in different $\mathrm{pH}$ environments, the drug will have different levels of ionization following the influence of the partition coefficient, as well as its permeation ability via the skin. Therefore, modification of the permeation parameters of SC has been reported as a major task in the development of an optimal drug for topical delivery formula; this includes improvement of onset time, maintenance of plasma level, and enhancement of skin permeation with subsequent lowering in dose and adverse effects. ${ }^{2,4}$ Yonessi and Saeedi ${ }^{3}$ investigated the effect of topical gel containing SC (1\% SC in different cosolvents) compared with SC oral tablets on erectile dysfunction. They reported a significantly shorter onset of action and lower side effects for transdermal SC delivery. However, only $12.5 \%$ of patients had complete erection with transdermal SC delivery, compared with $70 \%$ of patients receiving oral therapy. Yonessi and Saeedi ${ }^{3}$ suggested that several percutaneous absorption enhancers could enhance transdermal SC delivery; this appears to be a promising approach for treatment of erectile dysfunction. However, no published trials on SC delivery systems and transdermal application have emerged so far.

Realizing the obstacles of SC oral delivery, the development of SC drug delivery systems for improving drug characteristics and therapy becomes a substantial undertaking. The aim of the study outlined in this article was 
to improve SC delivery characteristics using nanocarriers. In a previous study, the present authors investigated self-nanoemulsifying drug delivery systems and nanoemulsions for this purpose. ${ }^{19}$ In the current study, formulation and full in vitro appraisal of SC-loaded SLNs and NLCs were performed. The developed nanoparticles were designed in the previous study to fit both oral and topical application, whereas in the current investigation transdermal permeation of the prepared nanocarriers via human skin were assessed and compared with different drug suspensions. Furthermore, the physical shelf stability of both nanocarriers was assessed.

\section{Materials and methods Materials}

SC was obtained from Sun Pharmaceutical Industries Ltd (Mumbai, India). Cetyl palmitate (CP) was obtained from Goldschmidt (Essen, Germany). Gattefossé (Lyon, France) kindly donated glycerol monolinoleate (Maisine ${ }^{\mathrm{TM}}$ 35-1 [M]). Polyoxyl 40 hydrogenated castor oil (Cremophor ${ }^{\mathbb{B}}$ RH 40 [CR]) was obtained from BASF (Ludwigshafen, Germany). Span ${ }^{\mathrm{TM}}$ 85 (hydrophilic-lipophilic balance $($ HLB $)=1.8$ ) was obtained from Sigma-Aldrich (St Louis, MO). Propylene glycol and Tween 80 were obtained from Al-Nasr Pharmaceutical Chemicals Co (Cairo, Egypt). All other chemicals used were of analytical grade.

\section{High-performance liquid chromatography}

For this study, the authors utilized a previously validated high-performance liquid chromatography (HPLC) methodology ${ }^{20}$ with minor modifications, as outlined in the authors' previous work. ${ }^{19}$ The HPLC instrument (Series 200; PerkinElmer Inc, Waltham, MA) was equipped with a reversed-phase $\mathrm{C} 18$ column $(25 \mathrm{~cm} \times 4.6 \mathrm{~mm}$; particle size $=5 \mu \mathrm{m})$. The isocratic mobile phase, $20 \mathrm{mmol} / \mathrm{L}$ monopotassium phosphate ( $\mathrm{pH} 4.7)$ :acetonitrile (25:75), was run at a flow rate of $1.0 \mathrm{~mL}$ per minute at room temperature and the column effluent was monitored by an ultraviolet detector set at $293 \mathrm{~nm}$. A $20 \mu \mathrm{L}$ volume of each sample was automatically injected into the analytical column. Samples were injected concurrently within the standard solutions injections. The system was also equipped with a 600 Series Link Interface operated by TotalChrom chromatography data system software (v 6.2; PerkinElmer Inc, Waltham, MA). The calibration curve of peak area against SC concentration was $\mathrm{Y}=862402.4 \mathrm{X}-42324.3$, under SC concentration of $0.5-9 \mathrm{mg} \%$. Retention time was 4.3 plus or minus 0.3 minutes $\left(\mathrm{R}^{2}=0.9956\right.$; limit of quantification $=0.7 \mu \mathrm{g} / \mathrm{mL}$; accuracy $=96.6 \%$ ).

\section{Preparation of SLNs and NLCs}

As a consensus in the literature, high-pressure homogenization (HPH) is considered the optimum method for SLN formation. This is ascribed to exploitation of low surfactant content and avoidance of organic solvent incorporation. ${ }^{10}$ Nevertheless, HPH results in high particle surface energy and lower stability. ${ }^{17}$ In the current study, SLNs and NLCs were prepared using a modified high-shear homogenization method, in an attempt to combine the privileges of $\mathrm{HPH}$ while circumventing high-pressure implications. Two methods of drug loading, during and after carrier formation, were compared. In the first method, drug was dispersed in the surfactant/cosurfactant mixture (CR, Span 85, propylene glycol) using sonication (Julabo sonicator, USR-3; Julabo Labortechnik GmbH, Seelbach, Germany). Dispersion was aided by heating in the shaking water bath at $70^{\circ} \mathrm{C}$ for 5 minutes. Afterwards, the wax (CP) was added to the blend and melting was pursued for 20 minutes at $70^{\circ} \mathrm{C}$. Water was heated separately to the same temperature and was then added to the component blend under high-shear homogenization at $3000 \mathrm{rpm}$, at $70^{\circ} \mathrm{C}$ for 5 minutes (T 25 digital Ultra-Turrax ${ }^{\circledR}$; IKA, Staufen, Germany). The hot emulsion formed was poured into a fourfold volume of cold, distilled water under high-shear homogenization at $7000 \mathrm{rpm}$. Homogenization was pursued in a cold-water bath for 30 minutes. The nanoparticle dispersion formed was then filtered through a $0.8 \mu \mathrm{m}$ membrane filter (diameter $25 \mathrm{~mm}$; Micro Filtration Systems, Dublin, CA).

In the second method, solid lipid and surfactant blend were mixed and heated to $70^{\circ} \mathrm{C}$ for 20 minutes. Distilled water heated to the same temperature was then added under high-shear homogenization at $3000 \mathrm{rpm}$, at $70^{\circ} \mathrm{C}$ for 5 minutes. The hot emulsion formed was poured into a fourfold volume of cold, distilled water under high-shear homogenization at $7000 \mathrm{rpm}$. The placebo nanoparticles formed were then passed through a $0.8 \mu \mathrm{m}$ membrane filter. After lipid nanoparticles were formed, drug was added quantitatively to SLN dispersion and sonicated for 15 minutes. As for NLCs, the liquid lipid (M) was mixed with the surfactant mixture. Dispersion was aided by heating in the shaking water bath at $70^{\circ} \mathrm{C}$ for 5 minutes. The same steps were then repeated for both methods (before and after carrier formation). For both SLNs and NLCs, two external phases (carbonate buffer [pH 10.3] and distilled water) were utilized and compared for their effect on nanoparticle characteristics. Composition of different SLN and NLC formulations is shown in Tables 1 and 2, respectively. 
Table I Composition of sildenafil citrate (SC)-loaded solid lipid nanoparticle formulations $(\% \mathrm{w} / \mathrm{v})^{\mathrm{a}}$

\begin{tabular}{lllllll}
\hline Formula & SC & CP & CR & Span $^{\text {TM }} 85$ & $\begin{array}{l}\text { Carbonate } \\
\text { buffer }\end{array}$ & Water \\
\hline Xa & 0.05 & 2 & 4 & - & - & 13 \\
Xb & 0.05 & 2 & 3 & 1 & - & 13 \\
XI & 0.05 & 2 & 2.6 & 1.4 & - & 13 \\
X2 & 0.075 & 2 & 2.6 & 1.4 & - & 13 \\
X3 & 0.1 & 2 & 2.6 & 1.4 & - & 13 \\
X4 & 0.075 & 2 & 2.6 & 1.4 & 13 & - \\
X5 $^{\text {b }}$ & 0.075 & 2 & 2.6 & 1.4 & 13 & - \\
X6 $^{\mathrm{b}}$ & 0.1 & 2 & 2.6 & 1.4 & 13 & - \\
\hline
\end{tabular}

Notes: aAll formulae contain I\% propylene glycol as cosurfactant; bdrug added after carrier formation.

Abbreviations: CP, cetyl palmitate; CR, Cremophor ${ }^{\circledR} \mathrm{RH} 40$.

\section{Particle size analysis}

Average particle size was measured using a laser diffraction particle size analyzer (Cilas 1064; Cilas, Orléans, France). SLN and NLC dispersions were diluted with distilled water to concentrations of $0.25 \%$ and $7 \%$, respectively. Samples were ultrasonicated for 3 minutes before measurement. Results were represented as mean particle size (duplicate) plus or minus standard deviation (SD).

\section{Entrapment efficiency determination}

The entrapment efficiency (EE) of the prepared SLNs and NLCs was assessed using Vivaspin ultracentrifuge tubes (Vivaspin ${ }^{\circledR} 6$ Centrifugal Concentrator, molecular weight cutoff [MWCO] 100,000; Vivaproducts, Inc, Littleton, MA). Nanoparticle dispersion $(3 \mathrm{~mL})$ was added to the centrifuge tube and centrifugation was carried out for 15 minutes at $4000 \mathrm{rpm}$. The filtrate was diluted (1:3) with distilled water, and $\mathrm{EE}$ was determined as a percentage through the following equation:

$$
\mathrm{EE} \%=\frac{\mathrm{Mi}-\mathrm{Mf} \times 100}{\mathrm{Mi}}
$$

Table 2 Composition of sildenafil citrate (SC)-loaded nanostructured lipid carrier formulations $(\% \mathrm{w} / \mathrm{v})^{\mathrm{a}}$

\begin{tabular}{llllllll}
\hline Formula & SC & CP & M & CR & Span $^{\text {TM }} \mathbf{8 5}$ & $\begin{array}{l}\text { Carbonate } \\
\text { buffer }\end{array}$ & Water \\
\hline $\mathrm{NI}$ & 0.05 & 1.4 & 0.6 & 2.6 & 1.4 & - & 13 \\
$\mathrm{~N} 2$ & 0.075 & 1.4 & 0.6 & 2.6 & 1.4 & - & 13 \\
$\mathrm{~N} 3$ & 0.1 & 1.4 & 0.6 & 2.6 & 1.4 & - & 13 \\
$\mathrm{~N} 4$ & 0.075 & 1.4 & 0.6 & 2.6 & 1.4 & 13 & - \\
$\mathrm{N} 5^{\mathrm{b}}$ & 0.075 & 1.4 & 0.6 & 2.6 & 1.4 & 13 & - \\
$\mathrm{N} 6^{\mathrm{b}}$ & 0.1 & 1.4 & 0.6 & 2.6 & 1.4 & 13 & - \\
\hline
\end{tabular}

Notes: aAll formulae contain $1 \%$ propylene glycol as cosurfactant; bdrug added after carrier formation.

Abbreviations: CP, cetyl palmitate; CR, Cremophor ${ }^{\circledast}$ RH 40; M, Maisine ${ }^{\mathrm{TM}}$ 35-I.
In this equation, $\mathrm{Mi}$ is the initial weight of $\mathrm{SC}$ and $\mathrm{Mf}$ is the weight of the free (unentrapped) drug detected in the filtrate. Samples were measured using the previously detailed HPLC method. Results were represented as the mean percentage (duplicate) plus or minus SD.

\section{Freeze-drying and reconstitution}

Nanoparticle dispersion $(15 \mathrm{~mL})$ was placed in $50 \mathrm{~mL}$ vials. Mannitol was added to the nanoparticle dispersion as a cryoprotectant in a final concentration of $4 \%(\mathrm{w} / \mathrm{v})$. The vials were frozen at $-80^{\circ} \mathrm{C}$ for 4 hours and were then placed in a lyophilizer (Cryodos-50; Telstar, SA, Terrassa, Spain) with a condenser temperature of $-70^{\circ} \mathrm{C}$. Lyophilization was performed at a pressure of $40 \mathrm{mbar}$ and a shelf temperature of $-40^{\circ} \mathrm{C}$ for 1 day, followed by a secondary drying at $25^{\circ} \mathrm{C}$ for another day. Lyophilized samples were kept at $-80^{\circ} \mathrm{C}$ until used. Reconstitution was carried out by manual redispersion of lyophilized samples in $15 \mathrm{~mL}$ of distilled water. Samples were then suitably diluted with distilled water (1:100) and particle size was measured using the laser diffraction particle size analyzer. Sonication was carried out for 3 minutes before measurement.

\section{Differential scanning calorimetry}

Differential scanning calorimetry (DSC) was employed to study thermal behavior and detect polymorphism of SLNs compared with NLCs. DSC thermograms of selected samples were obtained using a differential scanning calorimeter (PerkinElmer, Rodgau, Germany). The selected samples were:

- $\mathrm{CP}$ heated to the same procedure temperature $\left(70^{\circ} \mathrm{C}\right)$ as SLN and cooled suddenly in the same way (cold-water bath)

- physical mixture of CP and M (CP:M PH MIX) in formulation ratio $(0.7: 0.3)$, prepared in the same way as the CP sample

- physical mixture of CP, M, and CR (CP:M:CR PH MIX) in formulation ratio $(0.7: 0.3: 1.3)$, prepared in the same way as the $\mathrm{CP}$ sample

- freeze-dried SLN (zero cryoprotectant)

- freeze-dried NLC (zero cryoprotectant)

- $\mathrm{SC}$ powder (as received).

Samples $(10 \mathrm{mg})$ were weighed, hermetically sealed in an aluminum pan, and heated at a constant temperature increase rate of $5^{\circ} \mathrm{C}$ per minute over a temperature range of $20^{\circ} \mathrm{C}-200^{\circ} \mathrm{C}$. The melting point, enthalpies, and onset temperature of the transition observed were reported. 


\section{In vitro drug release}

The in vitro drug release was assessed using the dialysis bag method. ${ }^{21}$ Drug release from optimum SLN formulation was compared with optimum NLC formulation, placebo nanoparticle dispersions, and drug suspension. SC was suspended in carbonate buffer ( $\mathrm{pH} 10.3$ ) (nanoparticle external phase) yielding suspension with the same drug strength of nanoparticles $(0.1 \mathrm{~g} \% \mathrm{w} / \mathrm{v})$. Nanoparticle dispersions and drug suspension $(7.5 \mathrm{~mL})$ were added to the dialysis bag (Visking ${ }^{\circledR}$ 36/32, 27 mm, MWCO 12,000-14,000; Serva, Heidelberg, Germany). The bag was fixed to a $100 \mathrm{~mL}$ glassstoppered container holding $50 \mathrm{~mL}$ of citrate buffer (pH 5). The experiment was carried out in a thermostated shaking water bath set at $37^{\circ} \mathrm{C}$ and $100 \mathrm{rpm}$. A placebo nanoparticle experiment was carried out concurrently. All samples were measured using HPLC analysis. All experiments were performed in triplicate. Results are presented as mean percentage of release plus or minus SD.

\section{Transdermal permeation study}

\section{Skin collection and preparation}

Intact, full-thickness human skin was obtained from arm plastic surgery. The subcutaneous fatty tissue was removed from the skin using a scalpel and surgical scissors. The skin surface was cleaned with Ringer's solution and allowed to dry. Skin was completely covered by saline-immersed tissue, packed in aluminum foil, and stored in a polyethylene bag at $-20^{\circ} \mathrm{C}$. Human skin has been reported to be stable for 3-6 months under these conditions. ${ }^{22}$ All investigations were conducted in full compliance with the ethical principles of Mustafa El-Naggar Hospital (Semoha, Alexandria, Egypt).

\section{Sample preparation and Franz diffusion cell assembly}

Transdermal permeation of SC from the two nanocarriers developed was compared with that from drug suspensions in two nanocarrier external phases (carbonate buffer [pH 10.3] and water) using the same sample volume $(0.75 \mathrm{~mL})$ and final concentration $(0.1 \%)$. The applied sample volume had considered unified amount of drug in all formulations $(0.75 \mathrm{mg})$ to circumvent the concentration gradient effect on the rate of drug release.

Transdermal permeation of SC from the elaborated nanomedicines was assessed using a modified Franz diffusion cell assembly ${ }^{23}$ under nonocclusive conditions. The skin samples were mounted on modified Franz diffusion cells with a surface area of $3.14 \mathrm{~cm}^{2}$ and a receptor volume of $8.5 \mathrm{~mL}$. The skin was fixed so that the dermal side of the skin was exposed to the receptor fluid and the hypodermis remained in contact with the donor compartment. The receptor fluid consisted of citrate buffer solution ( $\mathrm{pH} 5$ ). After an equilibration time of 15 minutes at $32^{\circ} \mathrm{C}$, formulations were applied in the donor compartment through direct contact to the skin. Diffusion cells were placed in a thermostatically controlled shaking water bath (type 3047; Köttermann, Hänigsen, Germany). Diffusion cells were shaken at $100 \mathrm{rpm}$, while the temperature was maintained at $32^{\circ} \mathrm{C}$, simulating skin surface temperature. Sampling was performed at $0.5,1.5,5$, and 24 hours. At each point, a $3 \mathrm{~mL}$ sample was withdrawn and replaced with fresh buffer. All experiments were carried out in triplicate. The HPLC method was used to measure the concentration of SC permeated in skin effluent samples.

\section{Stability study}

Stability study of selected SLN and NLC dispersions was carried out for 6 months. The nanodispersions were packed in well-sealed amber glass bottles at $4^{\circ} \mathrm{C}$. Samples were taken each month and were analyzed for EE and particle size. In addition, nanodispersions were visually inspected for any physical instability (separation, aggregations, and so forth).

\section{Statistical analysis}

Statistical analysis of the results was carried out using Student's $t$-test $(P<0.05)$.

\section{Results and discussion}

CP is a lipid wax used in SLN preparation ${ }^{24}$ and is also well recognized in topical application of SLNs.$^{18}$ SLNs prepared with $\mathrm{CP}$ have successfully enhanced the oral bioavailability of nitrendipine via lymphatic targeting. ${ }^{25}$ Furthermore, CPbased SLNs exhibited high physical stability compared with mono- and triglycerides. ${ }^{26}$ Preliminary studies investigated drug solubility in different system components. Different surfactants, cosurfactants, oily phases, and buffer systems were investigated. ${ }^{19}$ The ingredient that exhibited higher drug solubilization was picked up for nanoparticle formation. $\mathrm{CR}$, propylene glycol, and $\mathrm{M}$ were chosen as the optimum surfactant, cosurfactant, and oily phase, respectively.

The influence of numerous factors on particle size and EE was investigated. Eight SLN formulations and six NLC formulations were prepared to study the effects of these factors on formula characteristics, as shown in Tables 1 and 2, respectively. Characterization of SLNs and NLCs was based on particle size analysis and EE assessment, as shown in Tables 3 and 4, respectively. The factors investigated included (1) ratio of CR to Span 85; (2) drug dosage influence; (3) 
Table 3 Characterization of sildenafil citrate (SC)-loaded solid lipid nanoparticles

\begin{tabular}{llll}
\hline Formula & $\begin{array}{l}\text { Mean particle } \\
\text { size }(\mu \mathrm{m})\end{array}$ & $\begin{array}{l}\text { SC entrapped } \\
(\mathbf{m g})\end{array}$ & EE (\%) \\
\hline $\mathrm{Xa}$ & $1.13 \pm 0.03$ & $38.60 \pm 1.13$ & $96.50 \pm 3.88$ \\
$\mathrm{Xb}$ & $0.54 \pm 0.01$ & $35.15 \pm 0.64$ & $87.75 \pm 1.59$ \\
$\mathrm{X}$ & $0.36 \pm 0.02$ & $36.10 \pm 1.55$ & $90.25 \pm 3.88$ \\
$\mathrm{X} 2$ & $0.33 \pm 0.07$ & $46.55 \pm 0.28$ & $77.58 \pm 0.47$ \\
$\mathrm{X} 3$ & $0.35 \pm 0.14$ & $46.76 \pm 0.06$ & $58.45 \pm 0.07$ \\
$\mathrm{X} 4$ & $0.34 \pm 0.40$ & $50.79 \pm 0.76$ & $84.64 \pm 1.26$ \\
$\mathrm{X} 5$ & $0.21 \pm 0.60$ & $73.02 \pm 0.42$ & $97.36 \pm 0.56$ \\
$\mathrm{X6}$ & $0.18 \pm 0.15$ & $96.65 \pm 0.49$ & $96.65 \pm 0.49$ \\
\hline
\end{tabular}

Note: Data presented as mean \pm standard deviation.

Abbreviation: $\mathrm{EE}$, entrapment efficiency.

$\mathrm{pH}$ of the nanoparticle external phase; and (4) method of drug loading.

\section{Ratio of CR to Span 85}

An initial attempt of lipid nanoparticle preparation involved studying different ratios of CR (as oil-in-water emulsifier; HLB $=15$ ) to Span 85 (as water-in-oil emulsifier; HLB $=1.8)$. Three ratios were compared: 100:0 $(\mathrm{Xa})$, 75:25 (Xb), and 65:35 (X1). As Table 1 shows, the use of oil-in-water surfactant only (Xa) resulted in microparticles rather than nanoparticles. The addition of a water-in-oil emulsifier $(\mathrm{Xb})$ resulted in a significant decrease in particle size $(540 \mathrm{~nm})$. Higher percentage of Span 85 (X1) resulted in further significant decrease in particle size $(360 \mathrm{~nm})$. This could be explained regarding the required HLB value for $\mathrm{CP}$ $(\mathrm{HLB}=10)$. Realizing that the average HLB of CR is 15 , large particle size of Xa $(1.13 \mu \mathrm{m})$ could be justified. The $\mathrm{HLB}$ of the surfactant blend in $\mathrm{Xb}$ is 11.7 (Equation 2), while the HLB of the X1 surfactant blend is 10.4. The closeness of the X1 HLB to the required HLB of the lipid could explain its smaller particle size compared with $\mathrm{Xb}$.

Table 4 Characterization of sildenafil citrate (SC)-loaded nanostructured lipid carriers

\begin{tabular}{llll}
\hline Formula & $\begin{array}{l}\text { Mean particle } \\
\text { size }(\mu \mathbf{m})\end{array}$ & $\begin{array}{l}\text { SC entrapped } \\
(\mathbf{m g})\end{array}$ & EE (\%) \\
\hline NI & $0.34 \pm 0.22$ & $37.39 \pm 1.14$ & $93.47 \pm 2.83$ \\
N2 & $0.30 \pm 0.14$ & $47.62 \pm 0.12$ & $79.35 \pm 0.20$ \\
N3 & $0.32 \pm 0.13$ & $48.80 \pm 0.22$ & $68.95 \pm 3.37$ \\
N4 & $0.28 \pm 0.05$ & $58.35 \pm 1.20$ & $95.83 \pm 2.00$ \\
N5 & $0.13 \pm 0.03$ & $73.80 \pm 0.14$ & $98.40 \pm 1.13$ \\
N6 & $0.10 \pm 0.21$ & $97.50 \pm 0.52$ & $97.5 \pm 0.52$ \\
\hline
\end{tabular}

Note: Data presented as mean \pm standard deviation.

Abbreviation: $\mathrm{EE}$, entrapment efficiency.
HLB $($ SAA blend $)=$ HLB $(\mathrm{CR}) \times \%(C R)+$ HLB

$$
(\operatorname{Span} 85) \times \%(\operatorname{Span} 85)
$$

(SAA $=$ surface active agent.) The elaborated surfactant blend ratio $(65: 35)$ was used for preparation and further optimization of SLNs and NLCs. The ratio of oil to solid lipid was fixed at 30:70 in all NLC formulations. This is the most common ratio in oil-loaded SLN formulations. No attempts to increase $\mathrm{M}$ ratio were adopted to avoid significant reduction in $\mathrm{CP}$ melting point, taking into consideration its original low melting point $\left(43^{\circ} \mathrm{C}-53^{\circ} \mathrm{C}\right)$.

\section{Drug dosage influence}

In order to study the effect of increasing drug dose on formula characteristics, three SLN formulations (X1, X2, X3) (see Table 1) and three NLC formulations (N1, N2, N3) (see Table 2) were prepared. The formulae comprised three drug amounts (50, 75, and $100 \mathrm{mg} \% \mathrm{w} / \mathrm{v})$ respectively. As Table 3 shows, the EE value of SLN formulae decreased significantly as the drug load increased. Increasing drug dose from 50 to $75 \mathrm{mg}$ resulted in an increase in the entrapped weight in both SLNs and NLC. Nevertheless, by increasing the dose from 75 to $100 \mathrm{mg}$, the entrapped drug amount in SLN formulae was fixed to almost the same value $(46.5 \mathrm{mg})$. This value is consistent with the sum drug solubility in all nanoparticle ingredients with their applied ratios. As for NLC formulations (Table 4), a slight increase in SC $\mathrm{mg}$ entrapped was noticed in N2 $(47.62 \mathrm{mg})$ or N3 $(48.80 \mathrm{mg})$ compared with their analogues X2 and X3, respectively. The drug amount exceeding solubility in ingredients is supposed to partition into the external phase (water), in which it is relatively more soluble than in solid lipid. The slight improvement could be ascribed to incorporation of $\mathrm{M}$ into the lipid matrix. As there was no significant improvement realized in EE through increasing the drug dose from 75 to $100 \mathrm{mg}$, the following factor effect was studied based on a SC dose of $75 \mathrm{mg}(\mathrm{w} / \mathrm{v})$.

\section{$\mathrm{pH}$ of the nanoparticle external phase}

In an attempt to increase the EE of drug-loaded SLN and NLC formulae, the use of a nanoparticle external phase with minimum drug affinity was investigated. The lowest drug affinity to the nanoparticle aqueous phase is supposed to permit higher drug accumulation inside lipid nanoparticles and on the shell. Therefore, drug solubility in different $\mathrm{pH}$ buffer systems was assessed. The buffers used were citrate buffer ( $\mathrm{pH} 5)$, phosphate buffer ( $\mathrm{pH}$ 7.4), and 
carbonate buffer ( $\mathrm{pH}$ 10.3). It was found that drug solubility decreased significantly as the $\mathrm{pH}$ increased. Drug solubility was lowest in carbonate buffer $(0.05 \mathrm{mg} / \mathrm{mL})$, followed by phosphate buffer $(0.09 \mathrm{mg} / \mathrm{mL})$, and was highest with citrate buffer $(2.25 \mathrm{mg} / \mathrm{mL})$. This could be explained by the basic functional group of the drug (NH-piperazine, $\mathrm{pKa}$ 8.7). The reverse relationship between sildenafil solubility and solvent $\mathrm{pH}$ is reported elsewhere. ${ }^{4}$ The buffer system with the lowest drug solubilizing capacity (carbonate buffer, $\mathrm{pH}$ 10.3) was selected for the next preparations of SLN (X4) and NLC (N4). The prepared formulations were compared with their analogues prepared with water as external phase (X2 and N2, respectively). For SLNs (see Table 3), elevation of external phase $\mathrm{pH}$ resulted in an increase in the amount of drug entrapped: X4 had $50.79 \mathrm{mg}$; X2 had $46.5 \mathrm{mg}$. A more pronounced and significant increase was noticed in NLC formulation N4 (58.35 mg), prepared with carbonate buffer, compared with N2 (47.62 mg) (see Table 4). The enhanced improvement in EE of NLCs compared with SLNs could be explained by the fact that the solubility of active ingredients in oils is generally higher than in solid lipids. For this reason, the higher loading capacity could be achieved through the development of NLCs. ${ }^{16,24}$ It is worth noting that the $\mathrm{pH}$ of 10.3 was selected based on the literature investigating the topical and sublingual absorption of SC. Lia and Chang ${ }^{2}$ have reported that the transport amount of $\mathrm{SC}$ had the highest permeation rate in nude mouse skin, at $\mathrm{pH} 8-11$. On the other hand, Wang et $\mathrm{al}^{7}$ have contended that a $\mathrm{pH}$ of 10.3 is optimum for sublingual permeation of SC. Therefore, the $\mathrm{pH}$ of 10.3 was selected as a compromise, to prepare nanoparticles that could be useful for both routes of administration. The alkaline buffer ( $\mathrm{pH} 10.3)$ was selected as carbonate/bicarbonate constituents, while borax buffer was avoided. This was ascribed to the safety, regarding oral route, of the former buffer compared with the latter. Carbonate buffer was reported to be safe for both oral and topical application. The acute oral median lethal dose (LD50) for carbonate buffer in rats is $2.8 \mathrm{~g} / \mathrm{kg}$, while the dermal LD50 in rats is $>3 \mathrm{~g} / \mathrm{kg}$. In addition, sodium carbonate has no or only a low skin irritation potential. ${ }^{27}$

\section{Method of drug loading}

The previous optimization steps have indicated optimum components, optimum formulation ratios, and optimum aqueous phase $\mathrm{pH}$. For this step, SLNs (X4) and NLCs (N4) with high EE (84.64\% and $95.83 \%$, respectively) and reasonable particle size (340 and $280 \mathrm{~nm}$, respectively) could be obtained. Nevertheless, although it combines many advantages, the high-shear homogenization method utilized in the study suffers from drug loss in the filtration step. This loss was confined to $20 \%$ plus or minus $1 \%$ of the labeled drug amount. In an attempt to decrease such loss, a different method of drug addition was assessed. The drug was alternatively loaded to nanoparticles after carrier formation and the filtration step, as explained earlier in the section on preparation of SLNs and NLCs. The SLN formulation X5 (Table 1) and the NLC formulation N5 (Table 2) were prepared exactly as X4 and N4, except that drug was added after the filtration step. The amount of SC incorporated was significantly increased when drug was added after carrier formation, for both SLNs and NLCs (Tables 3 and 4, respectively). Regarding SLNs, there was a significant increase in drug entrapped in X5 (73 mg) compared with X4 $(50 \mathrm{mg})$. A significant increase was also noticed in the NLC formulation N5 (73.6 mg) compared with N4 (58.35 mg). To test the improved EE observed with the second method of addition, a higher SC dose (100 mg) was used in both SLNs (X6) and NLCs (N6). It was found that the amount of drug entrapped was also significantly enhanced in both cases. SLNs with $96.65 \mathrm{mg}$ of SC entrapped and with a particle size of $180 \mathrm{~nm}$ could be prepared. Furthermore, the NLC formulation N6 with $97.5 \mathrm{mg}$ of SC entrapped and a particle size of $100 \mathrm{~nm}$ could be prepared. For formulae X5, X6, N5, and N6 prepared via drug addition after carrier formation, a smaller globular size than others may be attributed to the additional sonication step.

\section{DSC}

In order to circumvent interfering peak of water, nanoparticle dispersions were subjected to DSC in the lyophilized form. ${ }^{16}$ Selected samples were thermally analyzed in an attempt to compare thermal characteristics of SLNs and NLCs. Results are demonstrated in Figure 2 and Table 5. $\mathrm{CP}$ revealed two peaks, a very minor peak at $32.4^{\circ} \mathrm{C}$ and a major peak at $47.12^{\circ} \mathrm{C}$. Such a behavior of the solid lipid reflects two distinct polymorphic forms. From the literature reviews, the form with the lower melting point is attributed to $\alpha$-polymorphic form, whereas the latter is attributed to the most stable $\beta$-polymorphic form. The melting point of $\mathrm{CP}$ in both SLNs $\left(43.6^{\circ} \mathrm{C}\right)$ and NLCs $\left(42.5^{\circ} \mathrm{C}\right)$ is lower than that of bulk material $\left(45.6^{\circ} \mathrm{C}\right)$. While the melting point in NLCs was lower than SLNs by only one degree, the minor reduction in melting point reflects minor disturbance in the structure and crystallinity of $\mathrm{CP}$ through the addition of the 


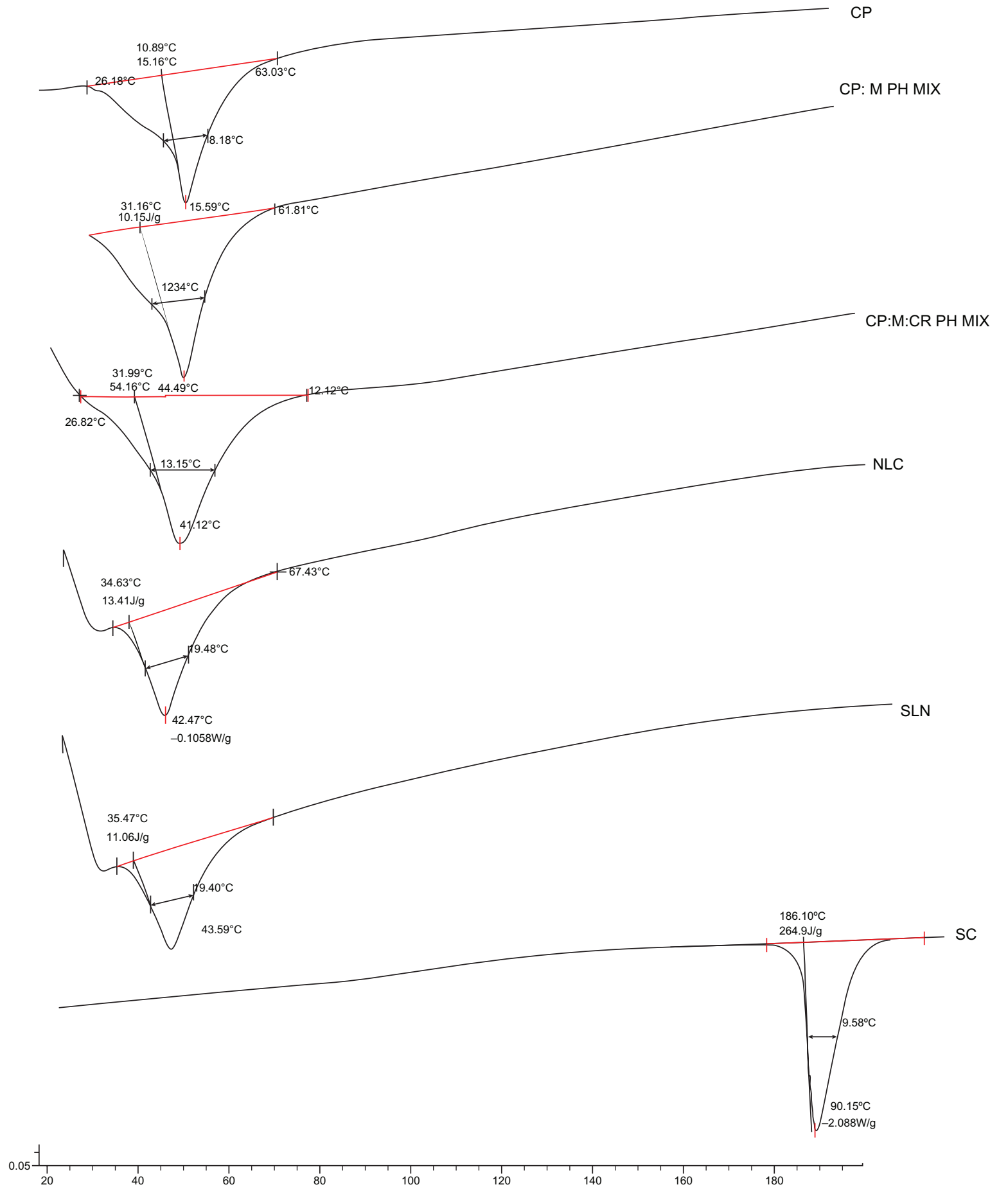

Figure 2 Differential scanning calorimetry thermograms of cetyl palmitate (CP); CP:Maisine ${ }^{T M}$ 35-I physical mixture (CP:M PH MIX) (0.7:0.3); CP:M:Cremophor ${ }^{\circledR}$ RH 40 physical mixture (CP:M:CR PH MIX) (0.7:0.3:1.3); nanostructured lipid carriers (NLCs), solid lipid nanoparticles (SLNs), and sildenafil citrate (SC).

applied ratio of oil. This was supported by a comparison of the melting point of $\mathrm{CP}\left(45.6^{\circ} \mathrm{C}\right)$ with that of the lipid:oil physical mixture $\left(44.5^{\circ} \mathrm{C}\right)$. These data explained and supported results of EE that demonstrated a minor difference in drug entrapped between SLNs and NLCs. This effect could be ascribed to the highly impacted crystalline structure of $\mathrm{CP}$ reported. ${ }^{26}$ It is worth noting that $\mathrm{SC}$ peak $\left(190^{\circ} \mathrm{C}\right)$ did not appear in either
SLN or NLC thermograms, owing to its very low percentage in the mixture $(0.83 \%)$.

\section{In vitro release study}

In the process of biological permeation, the drug should first release from the vehicles, and then it can be partitioned into or absorbed by the skin or gastrointestinal tract. ${ }^{11}$ 
Table 5 Peak onset, melting point, and enthalpy data of differential scanning calorimetry analysis

\begin{tabular}{lccr}
\hline Sample & \multicolumn{1}{l}{$\begin{array}{l}\text { Onset } \\
\left({ }^{\circ} \mathbf{C}\right)\end{array}$} & \multicolumn{1}{l}{$\begin{array}{l}\text { Melting } \\
\text { point }\left({ }^{\circ} \mathbf{C}\right)\end{array}$} & \multicolumn{1}{l}{$\begin{array}{l}\text { Enthalpy } \\
(\mathbf{J} / \mathbf{g})\end{array}$} \\
\hline CP & $40.89 \pm 0.08$ & $45.59 \pm 0.12$ & \multicolumn{1}{c}{$151.60 \pm 0.98$} \\
CP:M PH MIX & $34.46 \pm 0.07$ & $44.49 \pm 0.09$ & $107.50 \pm 0.85$ \\
(0.7:0.3) & & & \\
CP:M:CR PH MIX & $37.99 \pm 0.06$ & $44.92 \pm 0.14$ & $54.16 \pm 0.17$ \\
(0.7:0.3:I.3) & & & \\
SLNs & $35.47 \pm 0.33$ & $43.59 \pm 0.25$ & $11.06 \pm 0.16$ \\
NLCs & $34.63 \pm 0.15$ & $42.47 \pm 0.23$ & $13.40 \pm 0.12$ \\
SC & $186.70 \pm 0.49$ & $190.75 \pm 0.52$ & $264.90 \pm 0.63$ \\
\hline
\end{tabular}

Note: Data presented as mean \pm standard deviation.

Abbreviations: CP, cetyl palmitate; CR, Cremophor ${ }^{\circledR} \mathrm{RH} 40$; M, Maisine ${ }^{\mathrm{TM}}$ 35-I; NLCs, nanostructured lipid carriers; PH MIX, physical mixture; SC, sildenafil citrate; SLNs, solid lipid nanoparticles.

As a consequence, assessment of in vitro drug release is a crucial step in paving the way for good permeation. The in vitro release of SC from the selected SLN (X6) and NLC (N6) formulations was assessed using the dialysis bag method. As demonstrated in Figure 3, the release from both SLN and NLC dispersions was significantly higher than from drug suspension under the same experimental conditions. On the other hand, drug release from NLC formulation N6 was significantly higher than SLN formulation X6. These results are consistent with those obtained by Fang et al, ${ }^{16}$ who reported higher drug flux from NLCs than SLNs across an artificial membrane. They proposed that incorporation of liquid lipids into the solid lipid matrix caused the NLCs to become more imperfect, with the implication of easier drug release. In addition, the lower globular size of NLCs $(100 \mathrm{~nm})$, compared with SLNs (180 nm), may also participate in higher drug release.

It is worth noting that the lower particle size of NLCs $(100 \mathrm{~nm})$ compared with SLNs $(180 \mathrm{~nm})$ has been reported elsewhere. ${ }^{16}$ This could indicate better emulsification of the solid lipid matrix when oil is incorporated in the solid matrix. These results agree with findings from the authors' previous study ${ }^{19}$ where a nanoemulsion was prepared using $\mathrm{M}$ in the

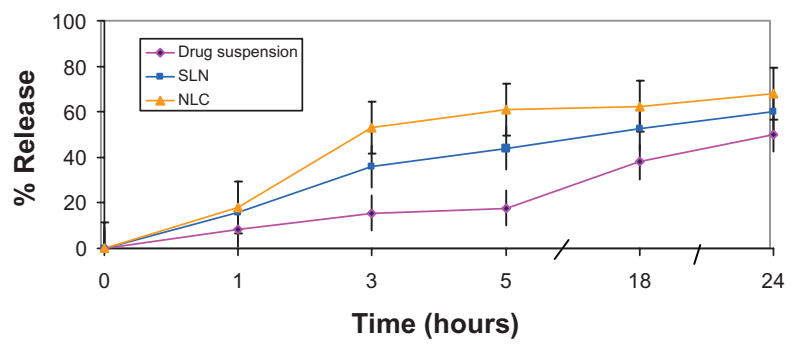

Figure 3 In vitro release profiles of sildenafil citrate from nanostructured lipid carriers (NLCs), and solid lipid nanoparticles (SLNs) compared with drug suspension in carbonate buffer ( $\mathrm{pH}$ I0.3) (citrate buffer, $\mathrm{pH}$ 5). oil blend and the same surfactant mixture of nanoparticles. The nanoemulsion exhibited further decrease in particle size $(70 \mathrm{~nm})$ compared with oil-loaded SLNs $(100 \mathrm{~nm})$ and SLNs themselves $(180 \mathrm{~nm})$.

The optimization steps resulted in preparation of SCloaded nanomedicines with promising in vitro characteristics. The ingredients utilized in SLN and NLC formulations are recognized for both oral and topical application. A future point of interest is the study of oral delivery of SC-loaded nanoparticles, considering incorporation of a bioactive surfactant-affecting cytochrome enzyme (CR), reported advantages of lipid nanoparticles in oral lymphatic targeting, and the capability of freeze-drying for preparation of nanocarriers as solid oral dosage form. ${ }^{9,28}$ In the current study, the transdermal permeation potential of the elaborated nanoparticles via human skin was assessed.

\section{Transdermal permeation study}

Excised human skin is properly regarded as the "gold standard" for in vitro penetration experiments related to human dermal risk assessment. Numerous animal skin models from various mammals and reptiles have been developed as surrogates for human skin..$^{29,30}$ Nevertheless, animal skin tends to be more permeable than human skin. ${ }^{23}$ Consequently, full-thickness human skin was utilized in the current study.

In order to investigate the effect of external-phase $\mathrm{pH}, \mathrm{SC}$ permeation levels from two different suspensions (carbonate buffer [pH 10.3] and water) were compared. As revealed in Figure 4, both suspensions exhibited low initial drug permeation (in the first 3 hours), which may be attributed to the formidable barrier of the stratum corneum. Subsequently, higher SC permeation was observed from the alkaline carbonate buffer than from the water suspension. This result is consistent with that obtained by Lia and Chang, ${ }^{2}$ who reported $\mathrm{pH}$-dependent permeation of $\mathrm{SC}$ via nude mouse skin. ${ }^{4}$ On the other hand, drug permeation rates from both

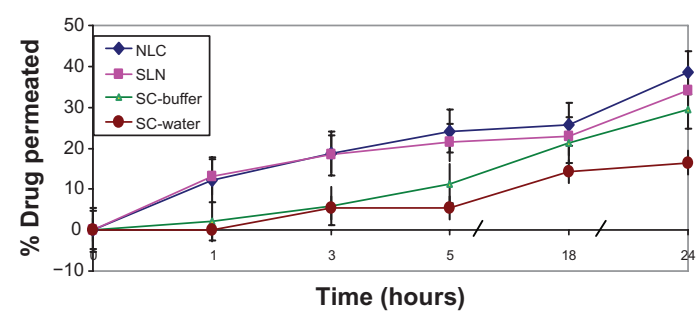

Figure 4 Transdermal permeation of sildenafil citrate (SC)-loaded nanoparticles across human skin versus drug suspensions in water and carbonate buffer $(\mathrm{pH}$ 10.3) (citrate buffer, $\mathrm{pH}$ 5)

Abbreviations: NLC, nanostructured lipid carrier; SLN, solid lipid nanoparticle. 
nanocarriers were significantly higher than from both drug suspensions, with significantly higher initial SC permeation and subsequent sustained release.

In the field of transdermal drug delivery, small particle size is implicated as large surface area and film formation that consequently creates intimate drug contact with the corneocytes. In this way, the nanosize of NLCs $(100 \mathrm{~nm})$ and SLNs $(180 \mathrm{~nm})$ is expected to increase the amount of encapsulated drug penetrating into the viable skin. ${ }^{12}$ Furthermore, the occlusive effect of lipid nanodispersions increases hydration of the stratum, which by reducing corneocyte packing and widening of the intercorneocyte gaps can facilitate drug penetration via skin. Occlusive effects appear strongly related to particle size; therefore, nanoparticles were reported to be significantly more occlusive than microparticles. ${ }^{18}$ An additional mechanism of enhancement involves the fact that surfactants can loosen or fluidize the lipid bilayers of the stratum corneum and thus act as permeation enhancers. ${ }^{16}$ It is worth noting that both SLNs and NLCs exhibited almost the same initial permeation profile, whereas higher SC permeation from NLCs than from SLNs was observed after 5 hours of application. This could be attributed to lower particle size and better drug release from the particles as manifested in dialysis experiment.

\section{Stability study}

As a general consensus, waxes such as $\mathrm{CP}$ have been reported to exhibit higher physical stability than glycerides, owing to highly ordered crystal packing. ${ }^{26,31}$ In view of the current study, both nanoparticle dispersions were stored in closed bottles at $4{ }^{\circ} \mathrm{C}$ in an attempt to investigate physical stability of SLNs and NLCs in liquid state. Particle size and EE data are shown in Table 6. Visual inspection revealed no aggregations or separation for SLNs or NLCs over the study period. Results revealed that both SLNs and NLCs kept their particle size, indicating good physical stability of

Table 6 Stability study of sildenafil citrate-loaded solid lipid nanoparticles (SLNs) and nanostructured lipid carriers (NLCs): selected formulations (X6, N6)

\begin{tabular}{|c|c|c|c|c|}
\hline \multirow{2}{*}{$\begin{array}{l}\text { Time } \\
\text { (months) }\end{array}$} & \multicolumn{2}{|l|}{ SLN (X6) } & \multicolumn{2}{|l|}{ NLC (N6) } \\
\hline & $\begin{array}{l}\text { Particle } \\
\text { size }(\mathrm{nm})\end{array}$ & EE (\%) & $\begin{array}{l}\text { Particle } \\
\text { size }(\mathrm{nm})\end{array}$ & EE (\%) \\
\hline 0 & $180 \pm 0.15$ & $96.65 \pm 0.49$ & $100 \pm 0.21$ & $97.50 \pm 0.49$ \\
\hline I & $183 \pm 0.23$ & $96.70 \pm 0.60$ & $98 \pm 0.40$ & $97.50 \pm 0.80$ \\
\hline 3 & $180 \pm 0.18$ & $96.30 \pm 0.38$ & $103 \pm 0.57$ & $97 \pm 1.20$ \\
\hline 6 & $187 \pm 0.42$ & $96 \pm 1.20$ & $95 \pm 0.85$ & $97 \pm 0.70$ \\
\hline
\end{tabular}

Note: Data presented as mean \pm standard deviation. Abbreviation: EE, entrapment efficiency. the particles. In addition, EE values were also unchanged; this reflects avoidance of drug expulsion in the formulations elaborated for the period of the study. Results are consistent with those obtained by Jenning and Gohla, ${ }^{26}$ who reported high physical stability of $\mathrm{CP}$, the wax utilized. In addition, avoidance of HPH is proposed to play a part in enhancing the stability of nanoparticles.

\section{Conclusion}

$\mathrm{SC}$ is a first-line treatment drug for erectile dysfunction. However, oral delivery of SC is hampered by various drawbacks including first-pass metabolism, numerous side effects, relatively short duration, and long onset of action. Furthermore, drug delivery formulation and transdermal application of SC is challenged by its amphoteric nature, low oil and water solubility, $\mathrm{pH}$-dependent characteristics, and poor membrane permeability. In the current investigation, two different types of SC-loaded nanocarriers were optimized to improve drug characteristics and transdermal permeation. SC-loaded NLCs and SLNs could be elaborated to possess excellent EE and particle size. The optimized nanoparticles exhibited in vitro drug release superior to that of drug suspension. Elaborated NLCs had smaller particle size and higher drug release than SLNs. Both nanocarriers exhibited excellent stability for the study period. SLNs and NLCs exhibited enhanced transdermal permeation of SC via human skin, with higher initial release and controlled permeation profile than drug suspension alone. The NLCs elaborated in this study did not exhibit the usual significant superiority over SLNs in EE and stability. This was attributed to the highly ordered crystalline structure of $\mathrm{CP}$, with minimum disruption of the structure upon addition of the small percentage of oily phase. Nanocarriers developed in this study have successfully improved SC transdermal permeation via human skin, with promising implications for shorter onset and longer duration of drug action. Furthermore, the formulated nanoparticles possess promising in vitro characteristics for oral and sublingual delivery, an area for future study.

\section{Disclosure}

The authors report no conflicts of interest in this work.

\section{References}

1. Tsertsvadze A, Yazdi F, Fink HA, et al. Oral sildenafil citrate (Viagra) for erectile dysfunction: a systematic review and meta-analysis of harms. Urology. 2009;74(4):831-836.

2. Lia J, Chang TW. Determination of transdermal sildenafil in nude mouse skin by reversed-phase high-performance liquid chromatography. J Chromatogr B Biomed Sci Appl. 2001;765(2):161-166. 
3. Yonessi M, Saeedi M. A Double-blind placebo-controlled evaluation of the effect of topical sildenafil on erectile dysfunction. J Appl Res. 2005;5(2):289-294.

4. Badwan AA, Nabulsi L, AL-Omari MM. Sildenafil citrate. In: Brittain HG, editor. Analytical Profiles of Drug Substances and Excipients. New York: Academic Press; 2001:372-376.

5. Shin HS, Bae SK, Lee MG. Pharmacokinetics of sildenafil after intravenous and oral administration in rats: hepatic and intestinal firstpass effects. Int J Pharm. 2006;320(1-2):64-70.

6. Nichols DJ, Muirhead GJ, Harness JA. Pharmacokinetics of sildenafil after single oral doses in healthy male subjects: absolute bioavailability, food effects and dose proportionality. Br J Clin Pharmacol. 2002;(53 Suppl 1):S5-S12.

7. Wang Y, Chow MS, Zuo Z. Mechanistic analysis of $\mathrm{pH}$-dependent solubility and trans-membrane permeability of amphoteric compounds: application to sildenafil. Int J Pharm. 2008;352(1-2):217-224.

8. Cevc G, Vierl U. Nanotechnology and the transdermal route: a state of the art review and critical appraisal. J Control Release. 2010;141(3): 277-299

9. Manjunath K, Venkateswarlu V. Pharmacokinetics, tissue distribution and bioavailability of clozapine solid lipid nanoparticles after intravenous and intraduodenal administration. J Control Release. 2005;107(2):215-228.

10. Müller RH, Radtke M, Wissing SA. Solid lipid nanoparticles (SLN) and nanostructured lipid carriers (NLC) in cosmetic and dermatological preparations. Adv Drug Deliv Rev. 2002;(54 Suppl 1):S131-S155.

11. Küchler S, Herrmann W, Panek-Minkin GG, et al. SLN for topical application in skin diseases: characterization of drug-carrier and carriertarget interactions. Int J Pharm. 2010;390(2):225-233.

12. Pardeike J, Hommoss A, Müller RH. Lipid nanoparticles (SLN, NLC) in cosmetic and pharmaceutical dermal products. Int J Pharm. 2009; 366(1-2):170-184.

13. Han F, Li S, Yin R, Shi X, Jia Q. Investigation of nanostructured lipid carriers for transdermal delivery of flurbiprofen. Drug Dev Ind Pharm. 2008;34(4):453-458.

14. Uner M, Wissing SA, Yener G, Müller RH. Skin moisturizing effect and skin penetration of ascorbyl palmitate entrapped in solid lipid nanoparticles (SLN) and nanostructured lipid carriers (NLC) incorporated into hydrogel. Pharmazie. 2005;60(10):751-755.

15. Puglia C, Filosa R, Peduto A, et al. Evaluation of alternative strategies to optimize ketorolac transdermal delivery. AAPS PharmSciTech. 2006;7(3):64.

16. Fang JY, Fang CL, Liu CH, Su YH. Lipid nanoparticles as vehicles for topical psoralen delivery: solid lipid nanoparticles (SLN) versus nanostructured lipid carriers (NLC). Eur J Pharm Biopharm. 2008;70(2): 633-640.

17. Hu FQ, Jiang SP, Du YZ, Yuan H, Ye YQ, Zeng S. Preparation and characteristics of monostearin nanostructured lipid carriers. Int J Pharm. 2006;314(1):83-89.
18. Schäfer-Korting M, Mehnert W, Korting HC. Lipid nanoparticles for improved topical application of drugs for skin diseases. Adv Drug Deliv Rev. 2007;59(6):427-443.

19. Elnaggar YS, El-Massik MA, Abdallah OY. Sildenafil citrate nanoemulsion vs self-nanoemulsifying delivery systems: rational development and transdermal permeation. Int $J$ Nanotechnol. 2011;8(8-9):749-763.

20. Shim HJ, Lee EJ, Jung YH, et al. Determination of a new phosphodiesterase $\mathrm{V}$ inhibitor, DA-8159, in plasma and urine by highperformance liquid chromatography. J Pharm Biomed Anal. 2002;30(3): 527-533.

21. ElnaggarYS, El-Massik MA, Abdallah OY. Self-nanoemulsifying drug delivery systems of tamoxifen citrate: design and optimization. Int $J$ Pharm. 2009;380(1-2):133-141.

22. Dragicevic-Curic N, Gräfe S, Gitter B, Winter S, Fahr A. Surface charged temoporfin-loaded flexible vesicles: in vitro skin penetration studies and stability. Int J Pharm. 2010;384(1-2):100-108.

23. Barbero AM, Frasch HF. Pig and guinea pig skin as surrogates for human in vitro penetration studies: a quantitative review. Toxicol in Vitro. 2009;23(1):1-13.

24. Teeranachaideekul V, Souto EB, Junyaprasert VB, Müller RH. Cetyl palmitate-based NLC for topical delivery of Coenzyme Q(10): development, physicochemical characterization and in vitro release studies. Eur J Pharm Biopharm. 2007;67(1):141-148.

25. Kumar VV, Chandrasekar D, Ramakrishna S, Kishan V, Rao YM, Diwan PV. Development and evaluation of nitrendipine loaded solid lipid nanoparticles: influence of wax and glyceride lipids on plasma pharmacokinetics. Int J Pharm. 2007;335(1-2):167-175.

26. Jenning V, Gohla S. Comparison of wax and glyceride solid lipid nanoparticles (SLN). Int J Pharm. 2000;196(2):219-222.

27. Organisation for Economic Co-operation and Development Screening Information Data Sets. Sodium carbonate. Boston: UNEP publications; 2002. Available from: http://www.inchem.org/documents/sids/sids/ Naco.pdf. Accessed October 18, 2010.

28. Zhuang CY, Li N, Wang M, et al. Preparation and characterization of vinpocetine loaded nanostructured lipid carriers (NLC) for improved oral bioavailability. Int J Pharm. 2010;394:179-185.

29. Godin B, Touitou E. Transdermal skin delivery: predictions for humans from in vivo, ex vivo and animal models. Adv Drug Deliv Rev. 2007;59(11):1152-1161.

30. Seto JE, Polat BE, Lopez RF, Blankschtein D, Langer R. Effects of ultrasound and sodium lauryl sulfate on the transdermal delivery of hydrophilic permeants: comparative in vitro studies with full-thickness and split-thickness pig and human skin. J Control Release. 2010;145(1): 26-32.

31. Radomska-Soukharev A. Stability of lipid excipients in solid lipid nanoparticles. Adv Drug Deliv Rev. 2007;59(6):411-418.
International Journal of Nanomedicine

\section{Publish your work in this journal}

The International Journal of Nanomedicine is an international, peerreviewed journal focusing on the application of nanotechnology in diagnostics, therapeutics, and drug delivery systems throughout the biomedical field. This journal is indexed on PubMed Central,

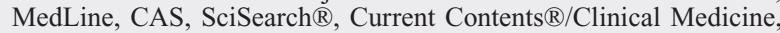

\section{Dovepress}

Journal Citation Reports/Science Edition, EMBase, Scopus and the Elsevier Bibliographic databases. The manuscript management system is completely online and includes a very quick and fair peer-review system, which is all easy to use. Visit http://www.dovepress.com/ testimonials.php to read real quotes from published authors. 